

\section{Estratificación socioeconómica y el desarrollo de las habilidades sociales en el estudiante}

\section{Socioeconomic stratification and social abilities development in the student}

\section{Saraí Aguilar Arriozola}

\section{Resumen}

Los estatus económicos y sociales tan variados, representados en los alumnos de los centros escolares son un reto actual. El propósito será relacionar las variables económicas con el desarrollo de habilidades sociales y mostrar la pertinencia del capital social como el potencializador del capital humano. El diseño de investigación se clasificó como aplicada con paradigma cuantitativo con una ejecución transversal y la obtención de información se llevó a cabo mediante la búsqueda en el campo de la realidad social, teniendo como resultado que el manejo de las habilidades sociales en los alumnos se da con diferencias significativas entre los miembros de los diferentes estratos. Por lo cual se concluye que es imposible lograr la optimización del recurso físico o de las habilidades humanas si no se impulsa el desarrollo de las habilidades sociales y se evita que los otros dos componentes de la triada se conviertan en limitantes de las habilidades sociales contenidas en el capital social.

Palabras clave: estratificación, estatus, habilidades, capital, investigación, desarrollo.

\section{Abstract}

The economic and social status represented in the students of school is a current challenge. The purpose will be to relate the economic variables with the development of social skills and show the relevance of social capital as the potential of human capital. The research design was classified as an applied research with a quantitative paradigm with a transversal execution and the obtaining of information was carried out through the search in the field of social reality, getting as a result that the management of social skills in students occur with significant differences between the members of the different strata. Therefore, it is concluded that it is impossible to achieve the optimization of the physical resources of human abilities unless the development of social skills is promoted and the other two components of the triad become limiting social skills contained in social capital.

Keywords: stratification, status, abilities, capital, investigation, development. 
on base a los intereses se encontraron trabajos de campo previos entre los que parece pertinente mencionar a Duek e Inda (2006), al examinar las ideas del sociólogo alemán Max Weber sobre la estratificación social, a la luz de los postulados centrales de su sistema teórico global. Por otra parte, de Sémbler (2006) es destacable su trabajo sobre estratificación social y clases sociales, ya que otorga una revisión analítica de los sectores medios e ilustra como la estratificación en América Latina va cobrando forma y se entienden los diferentes sectores sociales.

A Solís (2007) se le consideró en la lista de trabajos realizados meritorios de referenciar al estar ubicado espacialmente en la ciudad de origen de la población, Monterrey. Muestra como punto de partida la movilidad social y la estratificación desde un manejo estadístico y la evolución de la misma desde los años setenta hasta el 2008.

Se utilizará uno de los cuatro ejes manejados en la investigación, que es la que atañe: la estratificación social en la ciudad. Además, en su más reciente publicación habla de la transición de los alumnos de secundaria a nivel medio superior y la búsqueda de las causas de dicho abandono, entre las que estudia el factor social.

Si bien la investigación se abocará a los estudiantes que ingresan a pesar del contexto socioeconómico, pareció interesante el trabajo para ampliar el panorama al tener variables similares (estudiantes de bajo ingreso económico mismo nivel y el impacto social).
Bracho y Hernández (2009) argumentan en su estudio cómo vencer los retos presentados en la educación, con sociedades cada vez más polarizadas para que no se acentúen las diferencias, sino que sirva de instrumento para la integración social.

Con el interés de explorar posibilidades para aminorar el impacto de la estratificación social en el ambiente escolar, se ubicó la investigación realizada por Jiménez, Murga, Gil, Téllez y Trillo (2010) con alumnos de alto rendimiento, característica que asemejaba su población con la estudiada en esta investigación, donde una de sus principales interrogantes era conocer qué capacidades tienen chicos y chicas de alto rendimiento, cómo las utilizan y en qué manera influye el centro educativo en el desarrollo de las habilidades y capacidades integrales del estudiante.

Pareció de interesante consulta al es tar trabajando con una población similar. Asimismo Dayrell (2010) por su parte trabaja con las tres variables que se utilizan en esta investigación: juventud, socialización y escuela. Inscribió en una línea de estudios sobre las relaciones entre juventud y escuela, abordada desde el enfoque de las teorías de la socialización, entre muchos otros.

\section{Planteamiento del problema}

La estratificación social es reflejada y permeable en el estatus de los alumnos de preparatoria y la afectación del desarrollo de las habilidades sociales y conductas ante situaciones escolares. El alumno, como todo ser humano, trae consigo un conjunto de definiciones, que lo determina en sus interacciones en el ámbito escolar.

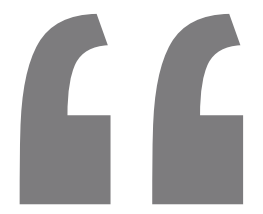

Bracho y

Hernández (2009)

argumentan en

su estudio cómo

vencer los retos

presentados en

la educación, con sociedades

cada vez más polarizadas

paraque no se acentúen las diferencias, sino que sirva de instrumento para la integración social". 
Una definición en el alumno es su acomodo en un determinado estrato de acuerdo al estatus socioeconómico que posea. Las habilidades sociales utilizadas por el alumno son herramientas claves para desarrollarse, desplazarse y enfrentar las situaciones escolares sociales.

En lo específico, la estratificación social a partir del contexto económico y su repercusión en el desarrollo de habilidades sociales es algo permeado al aula y al centro escolar. Existe una relación significativa entre las variables de origen (Estrato-estatushabilidades sociales).

El objetivo general de esta investigación es relacionar a través de la observación participante y la medición, las diferencias en las habilidades sociales en los miembros de una comunidad educativa a partir de la pertenencia a diferentes estratos socioeconómicos y del centro. Describir las diferencias y generar estrategias para subsanar las carencias en las habilidades sociales.

Los objetivos específicos de la investigación se exponen a continuación:

1. Contrastar el contexto socioeconómico de los estudiantes del muestreo.

2. Identificar las características presentes en los diferentes estratos sociales presentes en el Centro. 3. Describir las diferencias en las habilidades sociales a partir de la pertenencia a diferentes programas de bachillerato y su procedencia escolar.

4. Mostrar la pertinencia del capital social como el potencializador del capital humano.
5. Relacionar las variables económicas con el desarrollo de habilidades sociales.

La hipótesis que se plantea es la siguiente: La estratificación afecta el desarrollo social escolar del alumno. La justificación de la investigación es que el ser humano es netamente social. Desde que ingresa a este universo, lo hace a un mundo codificado en lenguaje, usos, costumbres, símbolos de los cuales es participe mediante la socialización con sus congéneres. Socialización en primera instancia con su núcleo familiar y en segunda, en la mayoría de los casos, con sus centros escolares.

Pero la sociedad en la cual está inserto, que une, que da identidad por derecho de pertenencia, por igual clasifica y da categorías a sus miembros no sólo a partir de sus similitudes, sino por diferenciación: gradándolos, generando estratos donde, a partir de las diferencias, se asignarán roles con diferente jerarquía en la escala social.

Esta jerarquización puede estar establecida sobre el sentido de posesión de bienes, habilidades, entre otras, genera códigos no escritos de comunicación entre los miembros de una misma sociedad pero de diferentes estratos.

Los miembros del mismo estrato parecieran generar conciencia de las diferencias más que de las similitudes, siendo no sólo éstas las causantes de la estratificación, sino esta conciencia la promotora de habilidades sociales diferentes entre los individuos perteneciente a cada estrato.

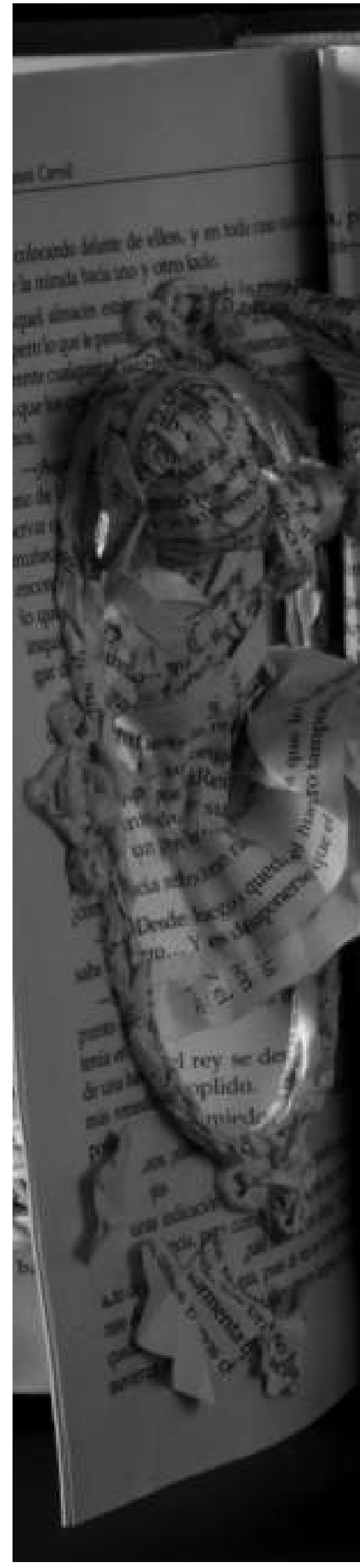

Presencia Universitaria 


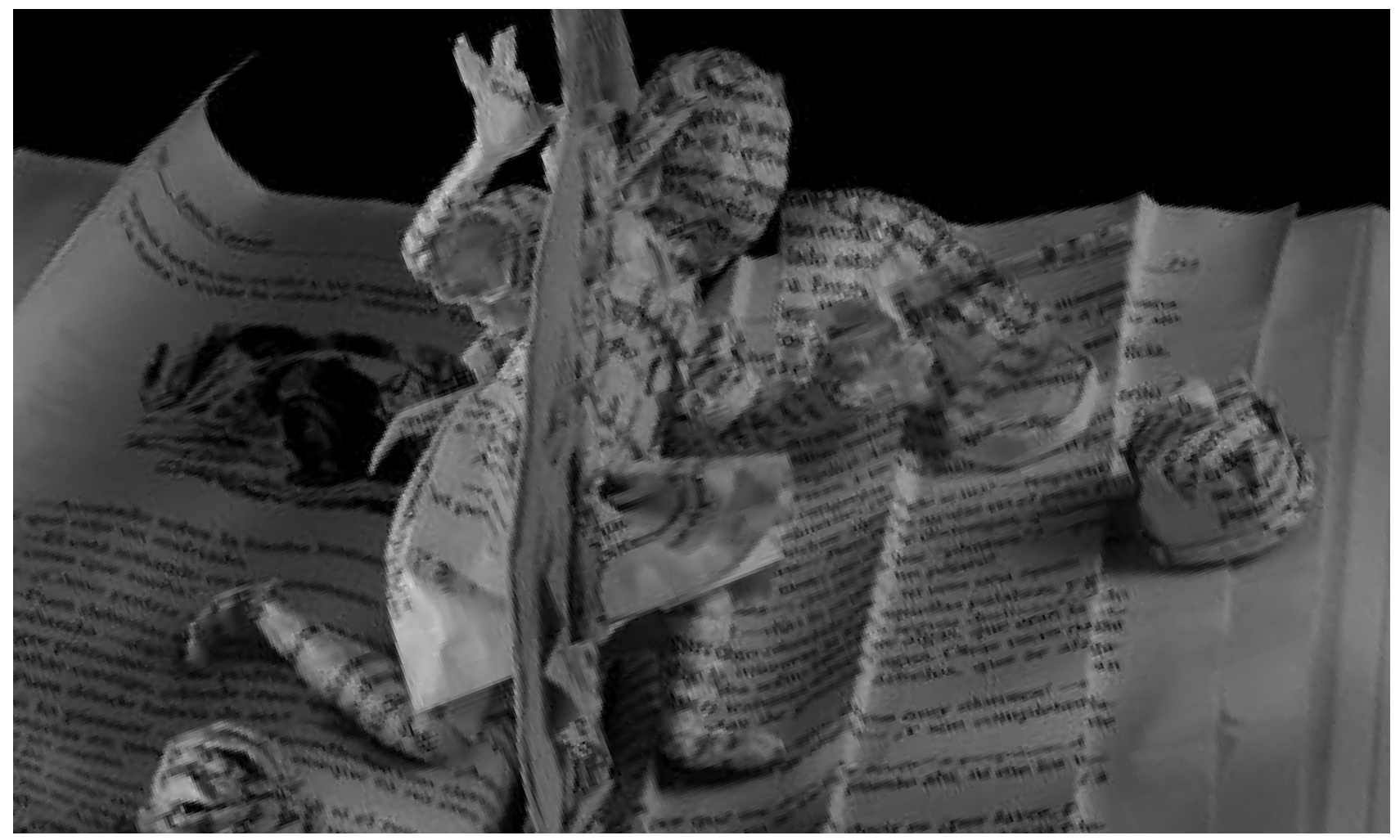

Los roles sociales no escritos pero si definidos, al parecer, son portables por el sujeto a los diferentes ambientes donde se desenvuelva; se tornan inherentes al mismo. Estas reflexiones llevan a cuestionar cómo maneja el ser humano en sus años adolescentes la estratificación social y económica en el centro escolar; más aún, si esta presencia afecta las habilidades sociales en su desarrollo y asertividad.

Estudiar, si como menciona Pérez Gómez (1995), la cultura pública, la académica, la social, la escolar y la privada, y cuyo conocimiento puede ayudar a clarificar el conjunto de factores plurales que condicionan los procesos de enseñanza-aprendizaje.

Es innegable que en estas últimas décadas se han aumentado las posibilidades de acceso a la educación media superior en el país. Las oportunidades de estudio se multiplican abriendo una gama de oportunidades. Al menos, ese es el discurso social que se ha repetido en numerosas ocasiones: a mayor educación, mayor posibilidad de reducir la brecha existente entre los diferentes miembros de la sociedad.

La educación, de acuerdo a lo enunciado por el Índice de Desarrollo Humano (IDH), de Amartya Sen y propuesto por el Programa de las Naciones Unidas para el Desarrollo (PNUD) para medir el nivel de desarrollo humano de un territorio, es considerada como una dimensión que da valor tangible a la calidad de vida.

El IDH se basa en los siguientes tres indicadores:

- Longevidad, medida en función de la esperanza de vida al nacer.
- Nivel educacional, medido en función de una combinación de la tasa de alfabetización de adultos (ponderación, dos tercios) y la tasa bruta de matrícula combinada de primaria, secundaria y superior (ponderación, un tercio). - Nivel de vida, medido por el producto interno bruto (PIB) real per cápita (paridad de poder adquisitivo, PPA en dólares).

Izquierdo (1979) menciona que la expectativa generada ante este cambio dista mucho de la evidencia que muestra; los cambios no se han visto reflejados en el desenvolvimiento integral de la nación.

Se puede mencionar, sin necesidad de profundizar, que el aumento del capital humano no ha redituado frutos en el físico, menos el social que es el que en esta investigación atañe. 
A la par, la desigualdad social, marcada en América Latina hace fácilmente observable la estratificación social con estatus delimitados, si bien por habilidades, carisma, en gran parte por posesión o no posesión de bienes. La triada conformada entre lo económico lo educativo y lo social es lo que se define en esta perspectiva.

La muestra está conformada por 200 adolescentes alumnos del Centro de Investigación y de Educación Bilingüe. Se utilizaron a maestros de los alumnos a quienes se les capacitó sobre el instrumento el cual se aplicó a manera de encuesta. La duración del ejercicio es de 30 minutos aproximadamente.

La población se conformó por una muestra estratificada con una po- blación cautiva. La edad de los participantes se encuentra ubicada entre los 15 y 16 años, siendo alumnos de primer semestre de preparatoria.

El instrumento de investigación cuenta con 2 cuartillas compuestas por 3 secciones. En la primera cuartilla, se presenta el instrumento, se solicitan los datos para caracterizar la muestra, tales como su edad, escuela de procedencia, (para ubicar si es de escuela pública o privada) municipio donde radica (sector socioeconómico), semestre en el centro y bachillerato en el que está inscrito.

En esta misma sección se dan las instrucciones generales para la resolución del mismo, y se les informa del uso que se dará a la información recabada.
En la segunda sección, que inicia en la primera cuartilla, se elaboró una escala de auto percepción de manejo de habilidades sociales, para lo cual se presentaron 40 actitudes $\mathrm{y} / 0$ respuesta ante ciertas situaciones sociales y el alumno tendrá que evaluar qué tan posible o no es que responda ante el estímulo de esa manera.

Esta sección se subdividió en cinco apartados, cada una correspondiente a diferentes habilidades debidamente documentadas.

El primer apartado está relacionado a las habilidades iniciales, que conciernen al contacto inicial con otras personas. El segundo apartado, se relaciona con las habilidades sociales avanzadas, en interacción con un grupo en realización de actividades.

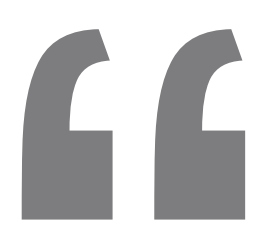

\author{
Los roles sociales no escritos pero si definidos, \\ al parecer, son portables por el sujeto \\ a los diferentes ambientes donde \\ se desenvuelva; se tornan inherentes al mismo".
}


El tercer apartado, es referente a los sentimientos y el manejo de ellos. El cuarto, sobre habilidades sociales en situaciones donde haya conductas agresivas en cualquier dirección. El quinto y último de la sección, es sobre el stress y la reacción ante él.

En la tercera sección, que abarca desde la segunda mitad de la tercera página hasta la cuarta página, se presentan poco más de una docena de preguntas para lograr caracterizar su estatus social y económico.

Las preguntas son simples y fundamentadas en el cuestionario proporcionado por Asociación Mexicana de Inteligencia de Mercado y Opinión Pública (AMAl), el índice de Niveles Socio Económicos (NSE).

La muestra está conformada por 200 adolescentes alumnos del Centro de Investigación y de Educación Bilingüe. Se utilizaron a maestros de los alumnos a quienes se les capacitó sobre la aplicación del instrumento el cual se aplicó a manera de encuesta. La duración del ejercicio es de 30 minutos aproximadamente.

Existen diferencias en el contexto económico de los alumnos del Centro. Se destacan alumnos procedentes de hogares donde los jefes de familia no cuentan con educación básica terminada y otros de hogares donde hay niveles de posgrado presentes.

Se encontraron notables contrastes en las características del capital físico de los alumnos por bachillerato. Se entiende por capital físico las posesiones materiales que permiten al humano ejercer su potencial en su plena expresión.

De tal manera que las características de las casas habitación muestran las diferencias en el contexto socioeconómico de los alumnos de diferentes bachilleratos.

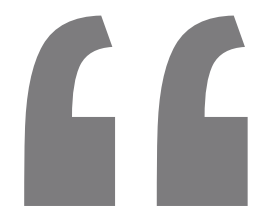

El manejo de las habilidades sociales en los alumnos se da con diferencias significativas entre los miembros de los diferentes bachilleratos. Esto se pudo contrastar mediante la aplicación de pruebas T para muestra independientes.

Las escuelas de procedencia (progresivo exclusivamente pública) de cada bachillerato están relacionados de manera significativa en el manejo y optimización de los alumnos de cada sistema más no así su lugar de procedencia.

las habilidades

sociales en los alumnos se da con diferencias significativas entre los miembros de los diferentes bachilleratos".
El principal hallazgo no es que las habilidades sociales sean bajas en sí, sino que en el manejo de las mismas, se hace un uso selectivo, siendo relevante mencionar que en los alumnos provenientes de escuelas públicas, con estratos sociales menos favorables que los alumnos provenientes de escuelas privadas, las habilidades relacionadas a la aceptación al rechazo, y las habilidades relacionadas con el manejo de reglas de cortesía, que lo hace notar como un alumno dócil, son las que se destacan. 


\section{Referencias}

Alcántara, H. T. R. (2011). Interacción social con confianza y apertura como factores que propician el aprendizaje en las organizaciones. Administración y Organizaciones, 14 (26), 2743.

Alcántara Santuario,A., y Navarrete Cazales, Z. (2014). Inclusión, equidad y cohesión social en las políticas de educación superior en México. Revista Mexicana de Investigación Educativa, 19(60), 213-239.

Barber B. (1964). Estratificación social. México: Fondo de Cultura Económica.

Becker, Gary S., (1983). El capital humano. Segunda Edición. España: Alianza Editorial, S.A. Pág. 15-251.

Bernardi, F.,y Cebolla,H.(2014). Clase social de origen y rendimiento escolar como predictores de las trayectorias educativas. Revista Española de Investigaciones Sociológicas (146), 3-21. doi: $10.5477 /$ cis/reis.146.3.

Boltvinik Kalinka, J. (1994). Pobreza y estratificación social en México. Instituto Nacional de Estadística, Geografía e Informática.

Bourdieu, P. (1998). Contra el fatalismo económico. New Left Review №. 227

Bracho, T., y Hernández, J. (2009). Equidad educativa: avances en la definición de su concepto. Presentada en el X Congreso Nacional de Investigación Educativa. Veracruz, México, Consejo Mexicano de Investigación Educativa.

C.Gómez Jaldón y J.A. Domínguez Gómez (2001). Sociología de la educación. Madrid: Pirámide. Callirgos, J. (2013). La discriminación en la socialización escolar. Biblioteca Virtual de Ciencias Sociales.

Castillo, J. C., Miranda, D., y Madero Cabib, I. (2013). Todos somos de clase media. Latin American Research Review 48(1), 155-173.

Dayrell, J. (2010). Juventud, socialización y escuela. Archivos de Ciencias de la Educación. Jiménez, C., Murga, M. Á., Gil, J. A., Téllez, J. A., y Trillo, M. P. (2010). Hacia un modelo sociocultural explicativo del alto rendimiento y la alta capacidad: ámbito académico y capacidades personales Revista: Educación XXI (13), 125-153.

Sémbler, C. (2006). Estratificación social y clases sociales: Una revisión analítica de los sectores medios (Vol. 125): United Nations Publications.

Solís, P. (2007). Inequidad y movilidad social en Monterrey. El Colegio de Mexico AC. 


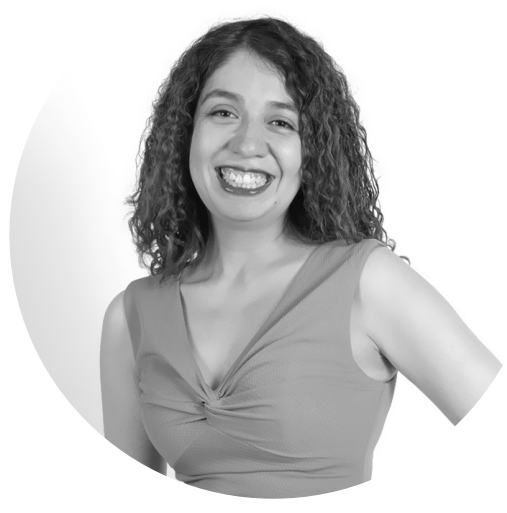

\section{Sarai}

\section{Aguilar Arriozola}

Doctora en educación por la Universidad Autónoma de Coahuila. Maestría en Artes con especialidad en Difusión Cultural por la Universidad Autónoma de Nuevo León y Licenciada en Educación por la Universidad Regiomontana. Cuenta con diversos diplomados sobre los derechos de las mujeres y el acceso a una vida libre de violencia por la Escuela virtual de Alta Formación de Derechos Humanos, y otros más en educación e investigación. Evaluadora para el International Baccalaureate Organization en el área de personas y sociedades. Coordinadora del Cuerpo Académico de Artes y Humanidades en el Centro de Investigación y Desarrollo de Educación Bilingüe de la Universidad Autónoma de Nuevo León. Enlace de Género del Instituto de Investigaciones Sociales de la Universidad Autónoma de Nuevo León para el Centro de Investigación y Desarrollo de Educación Bilingüe. Asimismo escribe editoriales semanalmente sobre género.

Correo electrónico:

arriozola2@gmail.com 